\title{
Ecosystem Integrity of the Upper Victoria Nile in East Africa based on Habitat and Fish Species Biotic Indices
}

\author{
S. Bassa ${ }^{1, *}$, A. Getabu ${ }^{2}$, D. O. Owiti ${ }^{3}$, A. M. Taabu ${ }^{4}$, E. Ogello ${ }^{3}$, N. E. Orina ${ }^{2}$, L. I. \\ Muhoozi $^{1}$, R. Olwa ${ }^{1}$, H. Nakiyende ${ }^{1}$, D. Mbabazi ${ }^{1}$, E. K. Muhumuza ${ }^{1}$, J. S. Balirwa ${ }^{1}$, W. \\ Nkalubo $^{1}$ \\ ${ }^{1}$ National Fisheries Resources Research Institute, Jinja, Uganda. \\ ${ }^{2}$ Department of Fisheries and Natural Resources, Maseno University, Kenya. \\ ${ }^{3}$ Department of Fisheries and Aquatic Studies, Kisii University, Kenya. \\ ${ }^{4}$ Lake Victoria Fisheries Organization, Uganda, Jinja, Uganda. \\ *Corresponding author. $\square$ 343, Jinja, Uganda @ sambasa37@yahoo.co.uk
}

\begin{abstract}
Riverine ecosystems are continuously been compromised by human activities resulting in threatening their integrity. In this study, integrity of Upper Victoria Nile River was assessed using habitat quality and fish biotic indices. Experimental gillnetting was done bi-annually in 9 stations along the river from 2008 to 2018 . Nine habitat metrics were used to estimate habitat quality index at every sampling station. Fish sampled, were sorted, identified to species level, and weighed. Counts of introduced and indigenous as well as tolerant and intolerant species were recorded to generate species richness. A total of 10.642 fish, 65 species belonging to nine families were recorded. Dominant species were Lates niloticus $62.79 \%$, Oreochromis niloticus $23.51 \%$, Mormyrus kannume $13.64 \%$; other species were $\leq 0.06 \%$. Tolerance and trophic guild showed carnivores $(61.5 \%)$, omnivores $(21.5 \%)$ and detrivores (16.9\%). Mean habitat quality index, total fish catch and fish-based index of biotic integrity varied among stations with highest record of $26.6 \pm 6.9,289.2 \pm 51.8$ and $30.6 \pm 7.9$ at sampling station (ST4) respectively. The lowest was $19.4 \pm 7.3,93.1 \pm 13.2$ and $26.7 \pm 6.8$ at sampling station (ST2) respectively. On a spatial basis, indices recorded significant differences among stations $(\mathrm{p}<0.05)$. Results indicated a fair fisheries biodiversity that need better conservation management of habitat type of the upper Nile.
\end{abstract}

Keywords: Habitat quality index, Fish- based index, Total fish catch.

\section{Introduction}

River Nile is the longest river in Africa, stretching from Uganda to the Mediterranean Sea. At 6800 kilometres, worldwide, it is second only to River Mississippi in length (Dumont, 2010). The river has important fisheries resources and plays a major role as spawning grounds for potamodromous fishes (Nkalubo et al., 2018) and as source of income for communities along the river (Dumont, 2010, NaFIRRI report, 2018). The Nile, including the upper part, has 
undergone a lot of modifications due to construction of hydropower dams and factories along its banks. In addition, agricultural activities have been done along the river. Along with intensive fishing exploitation, these developments have led to changes in habitats along the river and the fisheries in the ecosystem. Scanty work has been done in the investigation of the habitat quality and fisheries diversity and thus fish biotic index of the upper Nile. Hence, this study focused on the ecosystem of upper Victoria Nile, mainly on the habitat quality and fish biotic index in order to advise government and other stakeholders for proper sustainability of the fisheries.

The upper Victoria aquatic ecosystem status investigated through physical, chemical and biological approaches. It involved assessing the ecosystems parameters, habitat and fish abundances. This was geared at an approach of using habitat quality and aquatic biota in estimating the integrity of the aquatic ecosystem (Raburu and Masese 2010); this approach was referred to as bio monitoring. Effort has, been done to assess the integrity of riverine ecosystems for conservation and management using methods of physical-chemical parameters (Orina et al., 2018, Staniszewski, et al., 2006). Fish are increasingly becoming important in assessment of ecosystem integrity (Karr, 1981).

The fish biotic index has been used on Lake Victoria, Rivers Nzoa, Nyando, Sondu-Miriu and Kuja (Rabour and Manyala, 1990; Orina et al., 2018). Basing on the studies undertaken from other streams and rivers, upper Nile focused on undertaking habitat quality and fish biotic index in the Bujagali stretch. The main objective of this study was understudy the habitat along this stretch, the fisheries and water quality in order to advise the riparian people and other stakeholders. Through this study the local people along the shores of the upper Nile may be able to utilise both the fisheries and habitat type for sustainability and conservation.

\section{Materials and Methods}

\section{Study Area}

Sampling was conducted bi-annually at nine stations (ST1-ST9 i.e. $0.47524^{\circ} \mathrm{N}, 33.16699^{\circ} \mathrm{E}$ $0.512440^{\circ} \mathrm{N}, 33.129490^{\circ} \mathrm{E}$ ) in the UVN during the period 2008 to 2018 (Figure 1). Stations (ST1-ST3) have scanty islands in the transects gently sloping banks extensively cultivated with some tree cover of types Tremor orientalis and Ficus species and the margin with Vossia cuspidate macrophytes. Stations (ST4-ST6) located in the reservoir of the Bujagali hydropower dam with slow moving waters. Steel rolling mills are located about $3 \mathrm{~km}$ from these sites on the Buikwe side in addition to agricultural plantation of both sugarcane and other plants. Stations (ST7ST9) located in the downstream of the river below the Bujagali Dam with fast moving waters through rocky surface (Figure 1). 


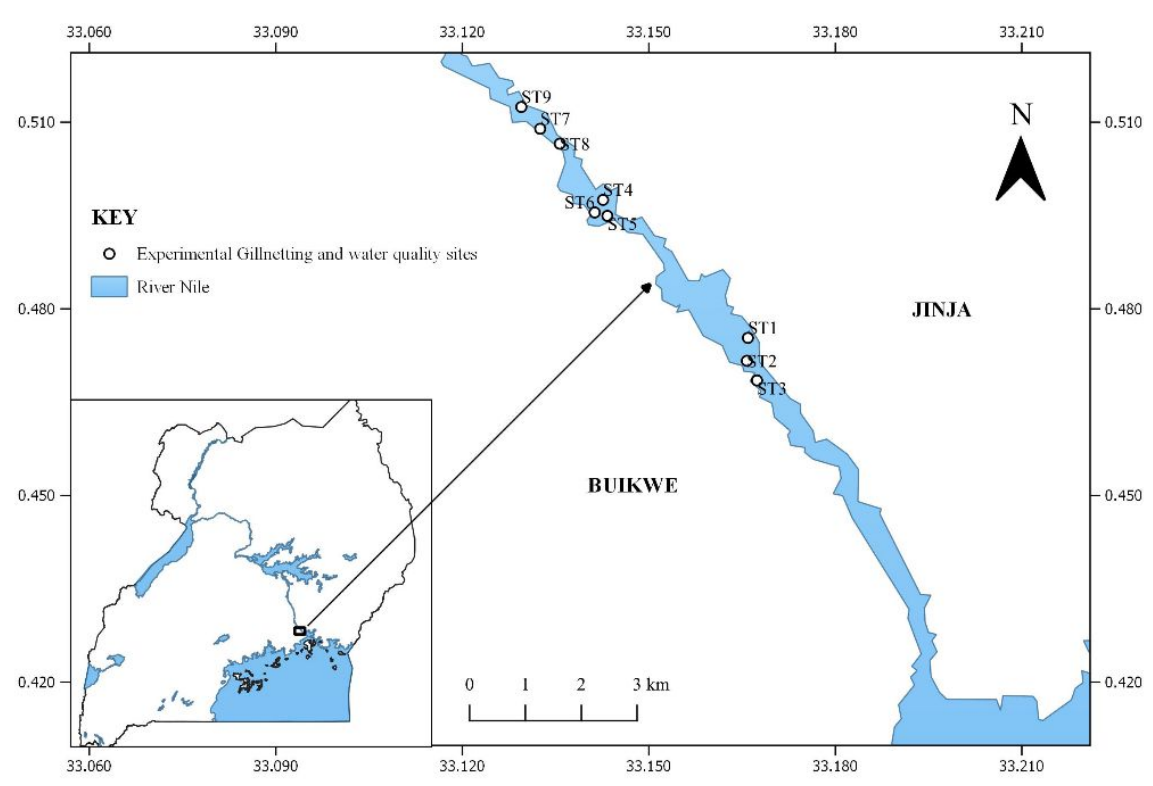

Figure 1. Map of upper Victoria Nile indicating the study sites (Inset: Map of Uganda)

\section{Habitat Quality Assessment}

A total of 9 metrics namely; river width, type of buffer vegetation, channel sinuosity, number of riffles, type of riverbed substrate, bank erosion profile, an aesthetic value, pool dimensions and available in stream cover of fish, were measured at each sampling station. The river width at each sampling station was calculated from GPS readings obtained at two positions on either bank of the river. The physical and chemical parameters; Dissolved oxygen (DO mg/L), temperature $\left(\mathrm{T}^{\circ} \mathrm{C}\right), \mathrm{PH}$, Total suspended solids TSS $(\mathrm{mg} / \mathrm{L})$ and conductivity Cond. ( $\mu \mathrm{Scm}$ 1) were measured in situ using a multi parameter $\mathrm{HacH} H \mathrm{HQ} 40 \mathrm{~d}$ Multiprobe. Nutrient sample measurements using a van dorn sampler collected in $500 \mathrm{ml}$ plastic bottles that were pre-cleaned, double rinsed with distilled water were immediately put in cooler boxes containing ice and transported to National Fisheries Resources Research Institute laboratory for analysis. Nutrient concentrations were determined from absorbance spectrophotometric measurement. This was done using modal UV Excellence UV/IVS spectrophotometer METTLER TOLEDO on pre filtered water samples using GFC filters of 0.45 microns (Apha 2005; Stantaiton, 1970). The nutrients levels determined were; Ammonia NH4-N (ug/L), Nitrate NO2-N ( $\mu \mathrm{g} / \mathrm{L})$, Nitrite NO3-N ( $\mu \mathrm{g} / \mathrm{L}$, Phosphate PO4-P ( $\mu \mathrm{g} / \mathrm{L})$, Total Phosphorus TP $(\mu \mathrm{g} / \mathrm{L})$, Total nitrate TN $(\mu \mathrm{g} / \mathrm{L})$ and Silicate SRSi $(\mu \mathrm{g} / \mathrm{L})$. These metrics used to estimate the HQI followed the criteria described by Raven et al., (1998) (Table 1). Using the estimated HQI the sampling stations were characterised from exceptional to minimal quality (Table 1). 
Table 1. Scoring Criteria for Habitat Characteristics

\begin{tabular}{|c|c|c|c|c|}
\hline \multirow{2}{*}{$\begin{array}{l}\text { Parameter } \\
\text { Available is } \\
\text { stream cover of } \\
\text { fish score }\end{array}$} & \multicolumn{4}{|c|}{ Scoring criteria } \\
\hline & $\begin{array}{l}\text { Abundant } \\
4\end{array}$ & $\begin{array}{l}\text { Common } \\
3\end{array}$ & $\begin{array}{l}\text { Rare } \\
2\end{array}$ & $\begin{array}{l}\text { Absent } \\
1\end{array}$ \\
\hline $\begin{array}{l}\text { Bottom substrate } \\
\text { score }\end{array}$ & $\begin{array}{l}\text { Stable } \\
>50 \% \text { gravel or } \\
\text { larger substrate }\end{array}$ & $\begin{array}{l}\text { Moderately stable } \\
30-50 \% \text { gravel or } \\
\text { larger substrate }\end{array}$ & $\begin{array}{l}\text { Moderately } \\
\text { unstable } \\
10-29.9 \% \text { gravel } \\
\text { or larger substrate } \\
2\end{array}$ & $\begin{array}{l}\text { Unstable } \\
<10 \% \text { gravel or } \\
\text { larger substrate } \\
1\end{array}$ \\
\hline $\begin{array}{l}\text { Dimension of } \\
\text { largest pool } \\
\text { score }\end{array}$ & $\begin{array}{l}\text { Large } \\
\text { Pool covers } \\
4\end{array}$ & $\begin{array}{l}\text { Moderate } \\
\text { Pool covers } \\
3\end{array}$ & $\begin{array}{l}\text { Small } \\
\text { Pool covers } \\
2\end{array}$ & $\begin{array}{l}\text { Absent } \\
\text { No riffles } \\
1\end{array}$ \\
\hline $\begin{array}{l}\text { Number of } \\
\text { Riffles score }\end{array}$ & $\begin{array}{l}\text { Abundant } \\
>5 \text { riffles } \\
4\end{array}$ & $\begin{array}{l}\text { Common } \\
2-4 \text { riffles } \\
3\end{array}$ & $\begin{array}{l}\text { Rare } \\
1 \text { riffles } \\
2\end{array}$ & $\begin{array}{l}\text { Absent } \\
\text { No riffles } \\
1\end{array}$ \\
\hline Water level score & $\begin{array}{l}\text { High } \\
<5 \% \text { if channel } \\
\text { substrate is } \\
\text { exposed } \\
3\end{array}$ & $\begin{array}{l}\text { Moderate } \\
\text { Water fills }>75 \% \\
\text { of the channel } \\
2\end{array}$ & $\begin{array}{l}\text { Low } \\
\text { Water fills } 25- \\
75 \% \\
1\end{array}$ & $\begin{array}{l}\text { No Flow } \\
\text { Very little } \\
\text { water in the } \\
\text { channel } \\
0\end{array}$ \\
\hline $\begin{array}{l}\text { Channel } \\
\text { sinuosity score }\end{array}$ & $\begin{array}{l}\text { High } \\
\geq 2 \text { well-defined } \\
\text { bends }\end{array}$ & $\begin{array}{l}\text { Moderate } \\
1 \text { well-defined } \\
\text { bend }\end{array}$ & $\begin{array}{l}\text { Low } \\
\text { Water fills } 25- \\
75 \%\end{array}$ & $\begin{array}{l}\text { No Flow } \\
\text { Very little } \\
\text { water in the } \\
\text { channel } \\
0\end{array}$ \\
\hline $\begin{array}{l}\text { Bank stability } \\
\text { score }\end{array}$ & Stable & Moderately stable & $\begin{array}{l}\text { Moderately } \\
\text { Unstable } \\
1\end{array}$ & Unstable \\
\hline $\begin{array}{l}\text { Riparian Buffer } \\
\text { Vegetation }\end{array}$ & $\begin{array}{l}\text { Extensive } \\
\text { Width of natural } \\
\text { Buffer is } \\
>20 \text { meters } \\
3\end{array}$ & $\begin{array}{l}\text { Wide } \\
\text { Width of natural } \\
\text { buffer is } 10.1-20 \\
\text { meters } \\
2\end{array}$ & $\begin{array}{l}\text { Moderate } \\
\text { Width of natural } \\
\text { buffer is } 5-10 \\
\text { meters } \\
1\end{array}$ & $\begin{array}{l}\text { Narrow } \\
\text { Width of } \\
\text { natural buffer is } \\
<5 \text { meters } \\
0\end{array}$ \\
\hline $\begin{array}{l}\text { Aesthetics of } \\
\text { reach score }\end{array}$ & $\begin{array}{l}\text { Wilderness } \\
\text { Outstanding } \\
\text { natural beauty }\end{array}$ & $\begin{array}{l}\text { Natural Areas } \\
\text { Trees or native } \\
\text { vegetation }\end{array}$ & $\begin{array}{l}\text { Common setting } \\
\text { Area is developed }\end{array}$ & $\begin{array}{l}\text { Offensive } \\
\text { Clutters; may } \\
\text { be a dumping } \\
\text { area } \\
0\end{array}$ \\
\hline
\end{tabular}

Source: (Raven et al., 1998; Orina et al., 2018)

\section{Fish-Based Index of Biotic Integrity (FIBI)}

Fish samples were obtained at each station using gillnets, mesh sizes of range 25.4 to $202.4 \mathrm{~mm}$ and beach seine $<25.4 \mathrm{~mm}$. To determine FIBI a total of 12 metrics namely native, intolerant, 
rheophilic, benthic, tolerant, cyprinidae, detrivores, carnivores, omnivores and exotic species, number of individual fish species per $50 \mathrm{~m}$ of sampling and modified fish index of wellbeing were determined (Table 2). Feeding guild status herbivores, omnivore and carnivore, were determined both visually and microscopically at x10 magnification using optical microscope in the field and laboratory respectively. Identification of fish to determine species diversity was done using keys in Witte and Van Densen (1995) and Rogers, 2016. FIBI was estimated using the metrics in (Table 2).

Table 2. Fish-Index of biotic Integrity (FIBI) metrics and its scoring criteria

\begin{tabular}{llccc}
\hline Category & Metric & \multicolumn{3}{c}{ Scoring criteria } \\
& & 1 (worst) & 3 & 5 (best) \\
\hline Species & No. of native species & $<3$ & $3-5$ & $\geq 6$ \\
richness and & No. Intolerant species & $<3$ & $3-5$ & $\geq 6$ \\
composition & No. Of rheophilic species & 0 & 1 & $>1$ \\
& \% of benthic species & $<7.5$ & $7.5-15$ & $>15$ \\
& \% of tolerant individual & $>20$ & $10-20$ & $<10$ \\
& \% of Cyprinidae individual & $<40$ & $40-80$ & $>80$ \\
Trophic & \% of detrivores individual & $<7.5$ & $7.5-15$ & $\geq 15$ \\
metric & \% of carnivores individual & $<1$ & $1-4.4$ & $\geq 4.5$ \\
& \% of omnivores individual & $>45$ & $20-45$ & $<20$ \\
Abundance & No. of individual per 50m of sampling & $<25$ & $25-50$ & $>50$ \\
and & No. of exotic species & $\geq 2$ & 1 & 0 \\
condition & Modified index of well being & $<1.25$ & $1.25-2.50$ & $\geq 2.50$ \\
\hline
\end{tabular}

Each metric was scored and a summation computed. The stations were then translated as excellent, good, fair, poor or very poor depending on whether it was within the range of 50$60,40-49,30-39,20-29$ and $<20$, respectively.

A final range of FIBI was extracted from the individual ranges. Biodiversity indices used as metrics in estimation of fish indices of biological integrity were calculated using the following formulae. The percentage proportion of and individual species was estimated using the formula:

$$
\%=\frac{\text { number of individual fish population }}{\text { total number of fish in a community }} \times 100
$$

The condition factor, that assessed the health status of fish, was estimated using the following formula:

$$
\mathrm{MIWB}=0.51 n \mathbf{N}+0.5 \ln \mathbf{B}+H N+H B
$$

Where;

In- Natural log, $\mathbf{N}$ - Number of fish individuals caught per unit distance sampled,

B- Biomass of fish individuals caught per unit distance excluding tolerant and exotic species, $H N, H B$ - Shannon-Wiener diversity index based on fish numbers and biomass respectively. MIWB- the modified index of wellbeing.

Information recorded regarding the number of species, biomass and abundance were used to estimate the fish species diversity (Flower and Cohen 1990; Krebs, 1999) at every station. Four 
biodiversity indices (Shannon-Wiener, Simpson, Species evenness and Species richness) were computed using the following formulas;

Shannon-Wiener's diversity index formula;

$\mathrm{H}=$
$-\sum_{i=I}^{S} p_{i} \operatorname{In}\left(p_{i}\right) .$.

Evenness index;

$E_{H}=\mathrm{H} / \mathrm{InS}$

Simpson index of diversity;

Simpson index of diversity $=1-\mathrm{D}$

$D=\sum_{i=I}^{s} P i^{2}$

$\boldsymbol{p} \boldsymbol{i}$ - the proportion of individuals calculated as abundance of individual species divided by total number of individuals in the community sampled

In - the natural log

$\boldsymbol{\Sigma}$ - the sum of all calculation

$\mathbf{S}$ - the number of species

$\mathbf{H}$ - the Shannon index of diversity

D - Simpson index.

Spatial and temporal differences in FIBI and HQI were determined using analysis of variance (ANOVA). Post hoc Turkey test was, used to group stations with similar indices. The statistical tests performed using SPSS 20.0 for Windows 7 professional. Then for Shannon wiener, Simpson, mean evenness, and species richness were evaluated using Excel program.

\section{Results}

The estimated HQI ranged from $28.00 \pm 2.51$ at ST9 to $19.42 \pm 0.33$ standard deviation SD at ST6 with an overall means $24.00 \pm 0.439$ SD the index depicted a fluctuation trend downstream (Figure 2). The study results indicated that there was a significant variation of HQI between sampled stations $(\mathrm{F}=4.356 ; \mathrm{p}<0.05)$. The mean FIBI ranged from $26.20 \pm 0.50$ at ST5 to $31.00 \pm 0.81$ at ST9 with the overall mean of $31.8 \pm 2.52$ (Figure 3). Significant differences occurred between FIBI at Stations ST2, ST4, ST7, ST8, ST9, ST1 and ST6 (F=13.0515; p < $0.05)$ while FIBI at stations ST3 and ST5, were $(p>0.05)$. 


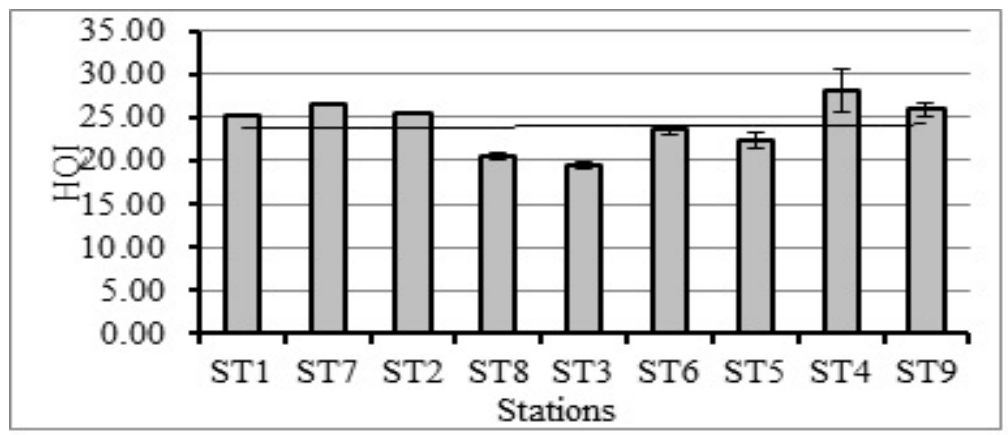

Figure 2. Mean Habitat quality index at sampling station upper Victoria Nile, Bujagali area

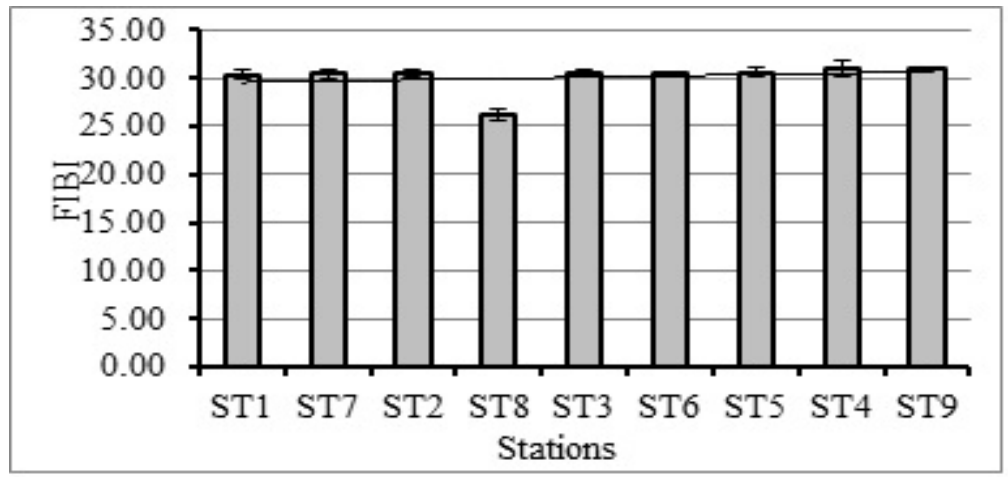

Figure 3. Mean FIBI at the sampling stations along upper Victoria Nile, Bujagali area

Ten thousand and six hundred forty two $(10,642)$ fish were recorded belonging to 9 families and 65 species. The families included the Cichlidae, Mormyridae, Latidae, Polypteridae, Mochokidae, Bagridae, Cyprinidae, Claridae and Alestidae. The fish species sampled, Oreochromis niloticus and Lates niloticus were introduced species while the rest were native. The most dominant were Lates niloticus $62.79 \%$, Oreochromis niloticus $23.51 \%$, Mormyrus kannume $13.64 \%$; other species were $\leq 0.06 \%$. The trophic guild indicated that Carnivores were more abundant $(61.5 \%)$, followed by omnivores (21.5\%) and Detrivores (16.9\%). There were no obligate herbivores in the stations sampled. The mean fish catch ranged from $654.14 \pm 126.49$ to $201.57 \pm 27.10$ at ST8 and ST2 respectively with the overall mean of $432.2 \pm 29.30$ (Figure 4).

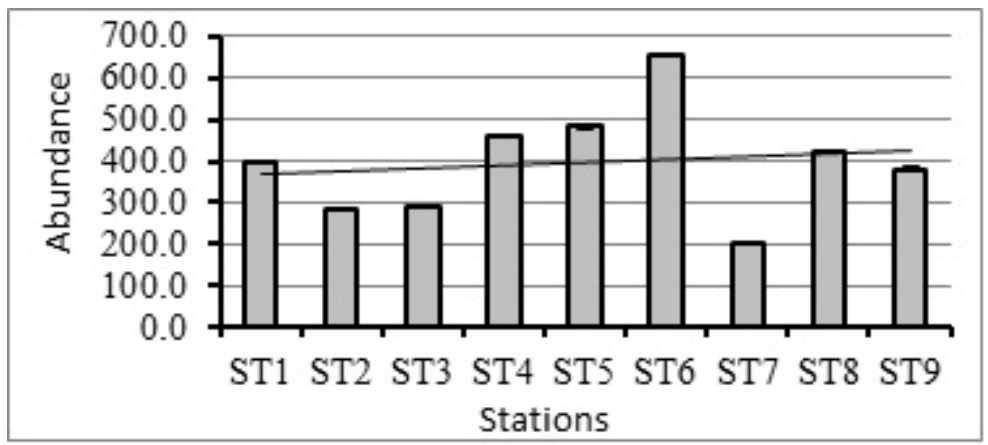

Figure 4. Mean abundance at the sampling stations upper Victoria Nile 
The spatial distribution indicated the mean catches declined downstream while its temporal distribution showed fluctuation. ANOVA on the mean catch rates indicated significant differences $(p<0.05)$ overtime the same applied to its spatial distribution. The Mean diversity indices, Shannon-Wiener index were $0.71 \pm 0.01$ in 2018 and $1.23 \pm 1.08$ in 2008, lowest to highest respectively. Simpson index ranged from $0.80 \pm 0.04$ in 2008 to $0.96 \pm 0.01$ in 2011 . Mean evenness index was low $0.14 \pm 0.00$ in 2017 and highest $0.22 \pm 0.00$ in 2018. Mean species richness index varied from $6.75 \pm 0.65$ in 2017 to $28.00 \pm 1.03$ in 2008 . The above indices revealed a general slight increasing trend however; the species Richness, Shannon and Simpson exhibited no significant variation between the months $(\mathrm{p}>0.05)$. It was only Evenness that had a significant variation of $F=5.178 ; \mathrm{p}<0.05$ for the whole period of sampling (Table 8). 
Table 3. The ecosystem parameters from the UVN from the sampling station (2008-2018)

\begin{tabular}{|c|c|c|c|c|c|c|c|c|c|}
\hline STATIONS & ST1 & ST2 & ST3 & ST4 & ST5 & ST6 & ST7 & ST8 & ST9 \\
\hline $\mathrm{DO}(\mathrm{mg} / \mathrm{L})$ & $5.7(0.1)$ & $5.5(0.2$ & $5.6(0.0)$ & $5.8(0.1)$ & $6.0(0.1)$ & $5.8(0.0)$ & $6.1(0.1)$ & $6.4(0.5)$ & $6.2(.0 .1)$ \\
\hline $\mathrm{T}^{\circ} \mathrm{C}$ & $25.7(0.1)$ & $25.6(0.2)$ & $26.2(0.0)$ & $25.7(0.2)$ & $25.9(0.1)$ & $25.9(0.2)$ & $26.2(0.0)$ & $25.8(0.4)$ & $25.8(0.2)$ \\
\hline $\mathrm{pH}$ & $7.3(0.1)$ & $7.2(0.1)$ & $8.2(0.2)$ & $7.3(0.2)$ & $7.5(0.2)$ & $7.3(0.2)$ & $7.5(0.0)$ & $7.4(0.1)$ & $7.3(0.1)$ \\
\hline Cond. ( $\mu \mathrm{Scm}-1)$ & $100.8(0.1)$ & $99.6(0.0)$ & $81.1(0.8)$ & $102.8(0.3)$ & $100.6(0.8)$ & $101.8(0.3)$ & $105.3(1.0)$ & $105.3(1.0)$ & $99.3(0.9)$ \\
\hline NH4-N (ug/L) & $2.3(0.1)$ & $2.9(0.2)$ & $2.4(0.4)$ & $3.3(0.7)$ & $3.8(0.1)$ & $3.4(0.7)$ & $3.1(0.0)$ & $3.1(0.0)$ & $3.0(0.1)$ \\
\hline NO2-N $(\mu \mathrm{g} / \mathrm{L})$ & $3.4(0.2)$ & $4.1(0.2)$ & $4.0(0.3)$ & $4.0(0.7)$ & $4.0(0.4)$ & $2.3(1.1)$ & $3.6(0.2)$ & $3.6(0.4)$ & 64(11.9) \\
\hline NO3-N ( $\mu \mathrm{g} / \mathrm{L})$ & $57.2(0.0)$ & $164.9(3.3)$ & $146.3(16)$ & $67.2(27.9)$ & $67.1(23.4)$ & $67.2(37.9)$ & $67.6(17.1)$ & $126(23.7)$ & $17.1(0.6)$ \\
\hline PO4-P ( $\mu \mathrm{g} / \mathrm{L})$ & $16.6(3.7)$ & $16.7(0.6)$ & $11.7(0.7)$ & $12.9(0.0)$ & $12.9(1.0)$ & $12.9(1.6)$ & $12.9(0.7)$ & $16.1(0.6)$ & $15.2(2.0)$ \\
\hline $\mathrm{TP}(\mu \mathrm{g} / \mathrm{L})$ & $77.5(9.2)$ & $89.6(4.1)$ & $58.5(4.1)$ & $54.0(4.4)$ & 53.492.9) & $54.0(6.5)$ & $54.2(2.0)$ & $83.1(5.4)$ & $65.8(2.0)$ \\
\hline $\mathrm{TN}(\mu \mathrm{g} / \mathrm{L})$ & $1471.5(289.2)$ & $382.5(146)$ & $1935(215)$ & $1347.1(355)$ & $1347.1(215)$ & $1347(216.6)$ & $1347(135)$ & $659(245)$ & $1688(617)$ \\
\hline SRSi $(\mu \mathrm{g} / \mathrm{L})$ & $119.4(23.0)$ & $392.1(57)$ & $63.8(8.2)$ & $282.7(48.2)$ & $282.7(48.2)$ & $327.2(106)$ & $63.8(46.9)$ & $63.8(8.4)$ & $162.3(45)$ \\
\hline $\mathrm{TSS}(\mathrm{mg} / \mathrm{L})$ & $3.1(0.00)$ & $3.6(0.3)$ & $4.4(0.3)$ & $3.7(0.30$ & $3.7(0.5)$ & $4.4(0.5)$ & $4.4(0.2)$ & $4.4(0.3)$ & $3.5(0.2)$ \\
\hline
\end{tabular}

Table 4. Comparison of HQI in this study and that of other rivers and streams

\begin{tabular}{|c|c|c|c|}
\hline Author & Habitat & Range (\%) & Comments \\
\hline Present study & Upper Victoria Nile -Bujagali area (2008-18) & $7.76-10.61$ & Severely degraded \\
\hline Orinal et al., 2018 & $\begin{array}{l}\text { River Kuja; (Kegati to river mouth as from November } 2016 \\
\text { to August 2017) }\end{array}$ & $35.50-77.4$ & $\begin{array}{l}\text { From severely degraded to partially } \\
\text { degraded. }\end{array}$ \\
\hline Raburu and Masese(2010) & $\begin{array}{l}\text { Lake Victoria basin (R. Nzoia, Nyando and Sondu-Miriu Feb, } \\
\text { March and July 2004) }\end{array}$ & $22.00-60.5$ & $\begin{array}{l}\text { From severely degraded to } \\
\text { degraded. }\end{array}$ \\
\hline $\begin{array}{l}\text { Bio habitat And Century } \\
\text { Engineering }(2016)\end{array}$ & $\begin{array}{l}\text { Anne Arundel county (Magothy, Sevem and Salamanders - } \\
\text { 2015) }\end{array}$ & $61.31-98.01$ & $\begin{array}{l}\text { From degraded to minimally } \\
\text { degraded sites. }\end{array}$ \\
\hline Paul et al.(2003) & $\begin{array}{l}\text { Maryland wadable streams (Piedmont class, Coastal plain and } \\
\text { Highland class from } 1994 \text { - 2000) }\end{array}$ & $15.43-99.35$ & $\begin{array}{l}\text { From severely degraded to } \\
\text { minimally degraded sites. }\end{array}$ \\
\hline Diana et al. (2006) & $\begin{array}{l}\text { Southern Michigan (Huron and Raisin basin from } 1999 \text { - } \\
\text { 2000) }\end{array}$ & $33.30-79.3$ & $\begin{array}{l}\text { From severely degraded to partially } \\
\text { degraded }\end{array}$ \\
\hline
\end{tabular}


Table 5. Comparison of FIBI in the study and of other rivers and streams.

\begin{tabular}{llll}
\hline Author & FIBI area research & Range (0-5) & Comments \\
\hline This study & Upper Victoria Nile (Bujagali area-2008-2018) & $1.8-3.5$ & Very poor to fair \\
Orina et al. (2018) & River Kuja;Kegati - river mouth as from Nov. 2016 to (Aug. 2017) & $1.70-4.5$ & Very poor to good \\
Raburu and Masese (2010) & Lake Victoria basin (R. Nzoia, Nyando and Sondu-Miriu-Feb, March and July & $1.70-4.6$ & Very poor to good \\
& 2004) & $1.67-3.67$ & Very poor to fair \\
Biohabitat and Century & Anne Arundel county (Magothy, Sevem and Salamanders 2015) & $1.00-5.00 \quad$ Very poor to good \\
$\begin{array}{l}\text { Engineering (2016) } \\
\text { Paul et al. (2003) }\end{array}$ & $\begin{array}{l}\text { Maryland wadable streams (Piedmont class, Coastal plain and Highland class } \\
\text { from 1994 - 2000) }\end{array}$ & Southern Michigan (Huron and Raisin basin from (1999-2000) & $0.25-3.85 \quad$ Very poor to fair \\
Diana et al. (2006) &
\end{tabular}


Table 6. Fish species classification as per the taxa, status, tolerance and trophic guild

\begin{tabular}{|c|c|c|c|c|}
\hline Family & Species & Status & Tolerance level & Trophic guild \\
\hline \multirow[t]{47}{*}{ Cichlidae } & Astatochromis pink dorsal & native & Omnivore & Intolerant \\
\hline & Astatoreochromis alluaudi & native & Omnivore & Intolerant \\
\hline & Astatotilapia "red dorsum" & native & Omnivore & Intolerant \\
\hline & Astatotilapia brownie & native & Omnivore & Intolerant \\
\hline & Astatotilapia elongate & native & Omnivore & Intolerant \\
\hline & Astatotilapia nubile & native & Omnivore & Intolerant \\
\hline & Astatotilapia purple dorsum & native & Omnivore & Intolerant \\
\hline & Astatotilapia red anal & native & Omnivore & Intolerant \\
\hline & Astatotilapia scarlet anal fin & native & Carnivore & Intolerant \\
\hline & Astatotilapia spp & native & Omnivore & Intolerant \\
\hline & Astatotilapia yellow & native & Omnivore & Intolerant \\
\hline & Coptodon zillii & native & Omnivore & Tolerant \\
\hline & Curved head Neochromis & native & Omnivore & Intolerant \\
\hline & Gaurochromis & native & Carnivore & Intolerant \\
\hline & Haplochromines & native & Detrivore & Tolerant \\
\hline & Harpagochromis "shovel mouth" & native & Detrivore & Intolerant \\
\hline & Harpagochromis guiarti & native & Carnivore & Intolerant \\
\hline & Lipochromis microdon & native & Carnivore & Intolerant \\
\hline & Lipochromis pavidens & native & Carnivore & Intolerant \\
\hline & Lithochromis & native & Omnivore & Intolerant \\
\hline & Mbipia "blue" & native & Omnivore & Intolerant \\
\hline & Mbipiambipi & native & Omnivore & Intolerant \\
\hline & Mbipia red & native & Omnivore & Intolerant \\
\hline & Mbipia yellow & native & Omnivore & Intolerant \\
\hline & Neochromis elongate & native & detrivore & Intolerant \\
\hline & Neochromis greenwoodi & native & detrivore & Intolerant \\
\hline & Neochromis nigricans & native & detrivore & Intolerant \\
\hline & Neochromis rufocaudalis & native & detrivore & Intolerant \\
\hline & Neochromis thichlips & native & detrivore & Intolerant \\
\hline & Oreochromis leucostictus & native & detrivore & Tolerant \\
\hline & Oreochromis niloticus & exotic & detrivore & Tolerant \\
\hline & Oreochromis variabilis & exotic & Omnivore & Tolerant \\
\hline & Paralabidochromis "black para" & native & Detrivore & Intolerant \\
\hline & Paralabidochromisi geneopinis & native & Omnivore & Intolerant \\
\hline & Paralabidochromis red cribensis & native & Omnivore & Intolerant \\
\hline & Paralabidochromis rockcrebensis & native & Omnivore & Intolerant \\
\hline & Paralabidochromis scarlet anal & native & Omnivore & Intolerant \\
\hline & Paralabidochromi ssp & native & Omnivore & Intolerant \\
\hline & Paralabidochromis thicklip & native & Omnivore & Intolerant \\
\hline & Paralabidochromis yellow rock & & & \\
\hline & picker & native & Omnivore & Intolerant \\
\hline & Paralibidochromis victoriae & native & Omnivore & Intolerant \\
\hline & Prognathochromis shovelmouth & native & Omnivore & Intolerant \\
\hline & Psammochromis riponianus & native & Omnivore & Intolerant \\
\hline & Psammochromis ssp. & native & Carnivore & Intolerant \\
\hline & Ptyochromis sauvagei & native & Carnivore & Intolerant \\
\hline & Ptyochromis ssp & native & Carnivore & Intolerant \\
\hline
\end{tabular}


Pundamilia macrocephala Pundamilia pundamilia

Pundamilia riponianus

Pundamulia yellowfin

Purple dorsum

R.cribensis

Shovel mouth

X. phytophagus

X.pink dorsal

Yssichromis earthquake

Claridae Clarias gariepinus

Cyprinidae Rastrineobola argentae

Latidae Lates niloticus

Mormyridae Mormyrus kannume

Polypteridae Protopterus aethiopicus

Mochokidae Synodontis afrofischeri

Synodontis victoriae

Bagridae Bagrus docmac

Cyprinidae Barbus altianalis

Alestidae

$\begin{array}{lll}\text { native } & \text { Carnivore } & \text { Intolerant } \\ \text { native } & \text { Omnivore } & \text { Intolerant } \\ \text { native } & \text { Omnivore } & \text { Intolerant } \\ \text { native } & \text { Omnivore } & \text { Intolerant } \\ \text { native } & \text { Omnivore } & \text { Intolerant } \\ \text { native } & \text { Omnivore } & \text { Intolerant } \\ \text { native } & \text { Omnivore } & \text { Intolerant } \\ \text { native } & \text { Carnivore } & \text { Intolerant } \\ \text { native } & \text { Omnivore } & \text { Intolerant } \\ \text { native } & \text { Omnivore } & \text { Intolerant } \\ \text { native } & \text { Omnivore } & \text { Tolerant } \\ \text { native } & \text { Omnivore } & \text { Intolerant } \\ \text { exotic } & \text { Carnivore } & \text { Intolerant } \\ \text { native } & \text { Carnivore } & \text { Tolerant } \\ \text { native } & \text { Carnivore } & \text { Tolerant } \\ \text { native } & \text { Omnivore } & \text { Tolerant } \\ \text { native } & \text { Carnivore } & \text { Tolerant } \\ \text { native } & \text { Carnivore } & \text { Tolerant } \\ \text { native } & \text { Omnivore } & \text { Tolerant } \\ \text { native } & \text { Omnivore } & \text { Intolerant }\end{array}$


Table 7. Pearson correlation matrix on the Ecosystem parameters

\begin{tabular}{|c|c|c|c|c|c|c|c|c|c|c|c|c|c|c|c|c|c|c|c|}
\hline & $\begin{array}{l}\text { TD } \\
(\mathrm{m})\end{array}$ & $\mathrm{SD}(\mathrm{m})$ & $\mathrm{DO}(\mathrm{mg} / \mathrm{L})$ & $\begin{array}{l}\mathrm{T} \\
{ }^{\circ} \mathrm{C} \\
\end{array}$ & $\mathrm{pH}$ & $\begin{array}{l}\text { Cond. } \\
(\mu \mathrm{Scm}- \\
1)\end{array}$ & $\begin{array}{l}\text { Depth } \\
\text { profile } \\
(\mathrm{m})\end{array}$ & $\begin{array}{l}\text { NH4- } \\
\mathrm{N} \\
\text { (ug/L) }\end{array}$ & $\begin{array}{l}\text { NO3- } \\
\mathrm{N} \\
(\mu \mathrm{g} / \mathrm{L})\end{array}$ & $\begin{array}{l}\text { PO4-P } \\
(\mu \mathrm{g} / \mathrm{L}) \\
\end{array}$ & $\begin{array}{l}\text { TP } \\
(\mu \mathrm{g} / \mathrm{L})\end{array}$ & $\begin{array}{l}\mathrm{TN} \\
(\mu \mathrm{g} / \mathrm{L})\end{array}$ & $\begin{array}{l}\text { TSS } \\
(\mathrm{mg} / \mathrm{L}) \\
\end{array}$ & $\begin{array}{l}\text { sum } \\
\text { HQI }\end{array}$ & RICHNESS & Simpson & Shannon & evenness & FIBI abundance \\
\hline TD (m) & 1 & & & & & & & & & & & & & & & & & & \\
\hline $\mathrm{SD}(\mathrm{m})$ & -0.02 & 1 & & & & & & & & & & & & & & & & & \\
\hline $\mathrm{DO}(\mathrm{mg} / \mathrm{L})$ & -0.213 & -0.09 & 1 & & & & & & & & & & & & & & & & \\
\hline $\mathrm{T}^{\circ} \mathrm{C}$ & -0.085 & -0.07 & 0.109 & 1 & & & & & & & & & & & & & & & \\
\hline $\mathrm{pH}$ & 0.127 & 0.122 & -0.49 & 0.32 & 1 & & & & & & & & & & & & & & \\
\hline Cond. $(\mu \mathrm{Scm}-1)$ & -0.042 & -0.26 & 0.106 & 0.15 & -0.31 & 1 & & & & & & & & & & & & & \\
\hline Depth profile (m) & 0.356 & -0.14 & -0.11 & -0.2 & -0.2 & 0.052 & 1 & & & & & & & & & & & & \\
\hline NH4-N (ug/L) & 0.128 & -0.2 & -0.3 & -0.3 & 0.09 & -0.386 & 0.28 & 1 & & & & & & & & & & & \\
\hline NO3-N $(\mu \mathrm{g} / \mathrm{L})$ & -0.147 & 0.194 & 0.038 & -0.2 & -0.04 & -0.336 & -0.05 & 0.112 & 1 & & & & & & & & & & \\
\hline PO4-P $(\mu \mathrm{g} / \mathrm{L})$ & -0.141 & 0.122 & 0.166 & 0.14 & 0.14 & -0.573 & -0.14 & 0.092 & 0.349 & 1 & & & & & & & & & \\
\hline $\mathrm{TP}(\mu \mathrm{g} / \mathrm{L})$ & -0.012 & 0.062 & -0.32 & 0.19 & 0.32 & -0.311 & -0.14 & 0.071 & -0.022 & 0.479 & 1 & & & & & & & & \\
\hline $\mathrm{TN}(\mu \mathrm{g} / \mathrm{L})$ & -0.016 & -0.06 & 0.396 & 0.22 & 0.06 & -0.334 & -0.1 & -0.08 & -0.175 & 0.543 & -0.028 & 1 & & & & & & & \\
\hline $\mathrm{TSS}(\mathrm{mg} / \mathrm{L})$ & 0.132 & -0.19 & 0.184 & 0.38 & 0.13 & 0.072 & 0.01 & 0.026 & 0.032 & -0.134 & -0.186 & 0.125 & 1 & & & & & & \\
\hline sum HQI & -0.492 & 0.215 & 0.529 & 0.01 & -0.25 & 0.018 & -0.33 & -0.44 & 0.171 & 0.09 & -0.281 & 0.279 & 0.088 & 1 & & & & & \\
\hline RICHNESS & -0.437 & 0.057 & 0.101 & 0.14 & -0.04 & -0.047 & -0.33 & -0.38 & -0.071 & 0.256 & 0.159 & 0.232 & -0.378 & 0.427 & 1 & & & & \\
\hline Simpson & -0.002 & 0.03 & -0.09 & -0 & -0.06 & -0.005 & 0.01 & -0.15 & -0.054 & 0.045 & -0.011 & 0.05 & -0.232 & 0.27 & 0.494 & 1 & & & \\
\hline Shannon & -0.441 & -0.02 & 0.135 & 0.06 & -0.04 & -0.025 & -0.31 & -0.06 & 0.119 & 0.252 & 0.1 & 0.157 & -0.217 & 0.353 & 0.384 & 0.543 & 1 & & \\
\hline evenness & -0.498 & 0.006 & 0.194 & 0.07 & -0.08 & -0.035 & -0.36 & -0.21 & 0.034 & 0.285 & 0.083 & 0.221 & -0.206 & 0.483 & 0.488 & 0.551 & 0.757 & 1 & \\
\hline FIBI & -0.062 & 0.085 & 0.221 & 0.07 & 0.01 & -0.001 & -0.09 & -0.18 & 0.101 & 0.036 & -0.074 & 0.056 & 0.035 & 0.39 & 0.252 & 0.38 & 0.21 & 0.236 & 1 \\
\hline abundance & 0.279 & 0.011 & 0.013 & -0.1 & -0.14 & 0.062 & 0.24 & 0.09 & -0.085 & -0.136 & -0.074 & -0.06 & 0.109 & -0.148 & -0.234 & -0.144 & -0.297 & -0.359 & $0.01 \quad 1$ \\
\hline
\end{tabular}

Table 8. Mean values ( \pm SE) for diversity indices in different sampling years

\begin{tabular}{|c|c|c|c|c|c|c|c|c|c|c|c|}
\hline & 2008 & 2009 & 2010 & 2011 & 2012 & 2013 & 2014 & 2015 & 2016 & 2017 & 2018 \\
\hline RICHNESS & $28.00(1.03)$ & $27.33(1.20)$ & $17.16(0.58)$ & $25.00(0.37)$ & $34.12(0.69)$ & $19.12(0.61)$ & $23.75(0.65)$ & $20.71(0.78)$ & $23.50(1.16)$ & $6.75(0.65)$ & $21.85(0.51)$ \\
\hline Simpson & $0.80(0.04)$ & $0.83(0.02)$ & $0.92(0.00)$ & $0.96(0.01)$ & $0.83(0.01)$ & $0.91(0.02)$ & $0.95(0.00)$ & $0.73(0.16)$ & $0.84(0.01)$ & $0.93(0.00)$ & $0.85(0.02)$ \\
\hline Shannon & $1.23(1.08)$ & $1.09(0.05)$ & $1.00(0.09)$ & $1.69(1.98)$ & $0.75(0.06)$ & $0.71(0.05)$ & $0.75(0.08)$ & $0.77(0.02)$ & $0.65(0.01)$ & $0.69(0.01)$ & $0.71(0.01)$ \\
\hline Evenness & $0.21(0.01)$ & $0.25(0.01)$ & $0.22(0.00)$ & $0.16(0.01)$ & $0.21(0.01)$ & $0.16(0.00)$ & $0.21(0.00)$ & $0.16(0.01)$ & $0.15(0.00)$ & $0.14(0.00)$ & $0.22(0.00)$ \\
\hline
\end{tabular}


The mean values for the physicochemical parameters (Table 3); recorded $\mathrm{PH}$, temperature ranged between $7.23 \pm 0.11-7.87 \pm 0.04 \mathrm{SD}$ and $25.75 \pm 0.46^{\circ} \mathrm{C}-26.6 \pm 0.12^{\circ} \mathrm{C}$ SD respectively that exhibited a little fluctuation trend downstream. Mean values DO, electrical conductivity, TSS ranged between $\left(5.73 \pm 0.231 \mathrm{mgL}^{-1} \mathrm{SD}\right)$ to $\left(6.80 \pm 0.12 \mathrm{mgL}^{-1}\right),\left(82.03 \pm 0.08 \mu \mathrm{Scm}^{-1}\right)$ to $\left(107.20 \pm 1.03 \mu \mathrm{Scm}^{-1}\right), 3.05 \pm 0.00-4.42 \pm 0.53 \mathrm{mgL}^{-1}$ respectively. The means exhibited a general increasing trend downstream. Then the mean value TN $(\mu \mathrm{g} / \mathrm{L})$, Ammonia, phosphorous, NitrateNO3-N $(\mu \mathrm{g} / \mathrm{L})$ ranged between $856.31 \pm 146.26 \mu \mathrm{gL}^{-1}$ to $3,049.60 \pm$ $355.65 \mu \mathrm{gL}^{-1}, 2.07 \pm 0.05 \mu \mathrm{gL}-1$ to $3.45 \pm 0.36 \mu \mathrm{gL}^{-1}, 53.45 \pm 4.18 \mu \mathrm{gL}^{-194} .10 \pm 6.58 \mu \mathrm{gL}^{-1}$ and $59.67 \pm 0.00$ to $\mu \mathrm{gL}^{-1}-155.22 \pm 11.96 \mu \mathrm{gL}^{-1}$ respectively. The mean values indicated an increasing trend downstream of the river. Meanwhile the mean values of Phosphate PO4-P $(\mu \mathrm{g} / \mathrm{L})$ and $\operatorname{SRSi}(\mu \mathrm{g} / \mathrm{L}-1)$ ranged from $12.98 \pm 2.06 \mu \mathrm{gL}^{-1}-$ to $16.34 \pm 0.65 \mu \mathrm{gL}^{-1}$, and from $63.80 \pm 46.48$ $\mu \mathrm{gL}^{-1}$ to $327.20 \pm 106.08 \mu \mathrm{gL}^{-1}$ respectively. The mean values indicated an increasing trend downstream. Pearson correlation indicated that FIBI positively correlated with $\mathrm{PH}(\mathrm{p}<0.05)$, conductivity $(\mathrm{p}<0.05)$, No2-N $(\mathrm{p}<0.05)$, PO4-P $(\mathrm{p}<0.05)$, TSS $(\mathrm{p}<0.05)$ and abundance $(\mathrm{p}<0.05)$ at a significant level of 0.05 . Then HQI correlated positively with Temperature $(\mathrm{p}<0.05)$, Conductivity $(\mathrm{p}<0.05)$ and abundance $(\mathrm{p}<0.05)$.

\section{Discussion}

This study provided baseline information on the indices that could be used for future assessment of HQIs on the upper Victoria Nile. Comparatively, the range of values of HQI of the UVN is narrower and lower than most of the HQI values for other rivers presented in the Table 4. The HQI values depicted a severely degraded ecosystem. This was supported by observations made on anthropogenic activities that included exposure of the river banks by farmers, deforestation, wetland degradation, urbanization, industrial pollution and hydropower dams. These have consequently resulted into structural alteration of the ecosystem integrity. The FIBI study values showed a poor to fair. The FIBI of the UVN ranges from very poor to fair and is compared with those other riverine in (Table 5). It is also narrower and slightly lower than those of other riverine ecosystem presented. It therefore confirms the observations of the HQI as indicated above that the UVN ecosystem is degraded.

The observed fluctuations of HQI and FIBI values along the UVN can also be due to complexity of the difference of ecosystems which the riverine habitat presents as described in methodology. The fluctuation nature is enhanced by continuous degradation of the UVN ecosystem due to anthropogenic activities. This justifies that habitats play a big role in bank stability, reduction of floods and organic matter accumulation and also alteration of the river with time and space (Ward, 1998; Orina et al., 2018; Raburu and Masese, 2010; Vannote et al., 1980; Johnson et al. 2001; Dumont 2010; Frank, 2014; Sitoki et al., 2010; Albinus et al., 2008).,

The 9 families and 68 fish species in the UVN indicated higher diversity of fish than found in Lake Victoria from which the river originates (Balirwa, 1998, Okaranon et al., 1999, Nkalubo et al, 2018). The trophic guild indicated that the introduced fish species Lates niloticus was dominant compared to the natives. The Carnivores were more abundant compared to Omnivores and Detrivores yet no obligate herbivores were recorded in terms of abundance though variations in numbers (Table 6 and 7). This indicates that Nile perch still takes high precedence in the population structure and diversity in the UVN. The decline of the catch rates downstream can be attributed to the fact that the water is fast flowing compared to reservoirs at Bujagali and Owen falls dam upstream where fishing is much easier and fishing crafts are not severely imbedded by the calm environment there. Further the changes in structure of the 
habitat downstream can also be the reason the catch rates declined. The downstream is habitat is constituted of deep rocky gorges with fast flowing water and this implies that only fish which can inhabit such ecosystem can be found there. One such rocky loving fish is Mormyrus kannume which was caught in large numbers in first running water than in other habitats (Bassa et al., 2018). The fish is known to scrape the rocky habitat to obtain its food as well as breed (Nkalubo et al., 2018). This suggests that the nature of the complexity of the habitat in the riverine UVN basin needs to be sustained in order to preserve the unique species occurred there. Most of the physical-chemical, PH. DO, TSS. Conductivity fall within tolerable levels compared to the main lake where the river originates. For example the $\mathrm{PH}$, DO, values from Lake Victoria were $7.8 \pm 0.2$ and $7.1 \pm 0.1 \mathrm{mg} / 1$ respectively (Olokotum, 2017). This could explain why the fish diversity was higher in the river than in the lake. Similar observations had been made by Sharma, et al. (2013); WHO, (2015); Orina et al. (2018); Chaurasia and Tiwari, (2012); and Sitoki et al. (2010).

\section{Conclusion}

The results indicated that the UVN had a diverse fish species with a high habitat quality interference. It justifies that human activities play a big role in retarding the ecosystem of the river. This led to fluctuations in dissolved oxygen, increased nutrient concentration, decreased habitat quality and fish community structure downstream. Then with the correlation analysis indicated that fish-based index of biotic integrity can be used to assess the habitat quality, abundance, and diversity of fish. Similarly, habitat quality index can be used to relate the fish species richness, abundance, and evenness index of biodiversity. Therefore, the integrity indices were largely dependent on the activities adjacent to the river.

\section{Recommendations}

There is need to improve, restore, maintain and constantly monitor ecosystem integrity so as to promote the sustainability of the riverine fisheries resources of UVN.

\section{Acknowledgement}

We thank the staff of the National Fisheries Resources Research Institute, Jinja, who were involved in the collection of data for the study. We also recognise the contribution and guidance of our great scientists and a colleagues, Okaranon John Obbo (RIP) and Musana Ali (RIP). We thank Bujagali Energy limited, Uganda, for funding this research.

\section{References}

Albinus M.P., Nakalle, J., Obando, J., and Y., Bamutaze, 2008. Effects of land use practices on livelihoods in the transboundary sub-catchments of the Lake Victoria basin. African journal of environmental science and Technology.vol.2 (10), 309-317.ISSN1996-0786. 
Apha, G. A.E., Lenore S.C., Eaton A.D., Awwa, 2005. Standard methods for examination of water and wastewater.18th edition. Prepared and published jointly by American public health association, American water Works Association, Water Environment federation.

Balirwa, J.S., 1998. Lake Victoria wetlands and the ecology of the Nile tilapia, Oreochromis niloticus Linne. PhD dissertation. Wageningen Agricultural University, Wageningen, The Netherlands.

Bassa S, D.O. Owiti, A. Getabu, H. Nakiyende, W. Nkalubo, J.S. Balirwa, V. Natugonza, D. Mbabazi and A.M. Taabu. 2018. Effects of exploitation pressures and river damming on the population structure of Elephant snout fish (Mormyrus kannume) Forsskal 1775: A case study on the upper Victoria Nile, East Africa. Uganda Journal of Agricultural Sciences, ISSN: 1026-0919 (Print); 2410-6909 (Online), Volume 18 Number 1 (2018) pp. 1 - 17.

Chaurasia, N. K., \&Tiwari, R. K. 2012.Physico-chemical characteristics of sugar factory and Distillery effluents. Annals of Biological Research, 3(9), 4406-4408.

Dumont, H.J. 2010.Descriptions of the Nile basin. Monographiae Biologicae; The Nile origin, environments, limnology and human use vol. 89, 1-2.Springer publishers.

Franklin, P.A. 2014. Dissolved Oxygen Criteria for Freshwater Fish in New Zealand: A Revised Approach, New Zealand Journal of Marine and Freshwater Research, 48:1, 112-126, DOI: 10.1080/00288330.2013.827123.

Johnson, D. H., Pimman, N., Wilder, E., Silver, J. A., Plotnikoff, R.W., Mason, B.C., Barreti, C., 2001. Inventory and monitoring of Salmon habitat in the Pacific Northwest: directory and synthesis of protocols for management/ research and volunteers in Washington, Oregon, Idaho, Montana and British Columbia. Washington department of fish and Wildlife, Oympia, Wa. 212 pp.

Karr, J. R. 1981. Assessment of Biotic Integrity Using Fish Communities. Fisheries, 6, 21-27.

Nkalubo, W., Balirwa, J., Bassa, S., Muhumuza, E., Nsega, M. and Mageni, R. 2018. Fish breeding areas as a management tool for resources in Lake Victoria, East Africa. African journal of Tropical hydrobiology and Fisheries 16:1-9 2018.Lake Victoria Fisheries Organization.

Okaranon J.O, Muhoozi L.I. and Bassa S.1999. Current state of the Fish Stocks of Lake Victoria (Uganda). In: D. Tweddle and I.G. Cowx: Report on fourth FIDAWOG workshop for the Lake Fisheries Research Project (Phase II), held at Kisumu (Kenya), 16th - 20th August 1999. Technical Document No. 5, LVFRP/TECH/99/05.30-39.

Olokotum, M. 2017. The occurrence and elimination of cyanobacteria toxins in portable water in Uganda. Case of Gaba III and Walukuba water works University of Natural Resources and life Sciences (Boku), Vienna, Austria.

Orina, E. N., A., Getabu, R. Omondi and E. Sigel 2018. Ecosystem integrity indices based on Fish diversity and physicochemical parameters in River Kuja, Kenya. Journal of Tropical hydrobiology and Fisheris.1:07-13.

Raburu, P. O. and Masese, F. O. 2010.Development of a Fish-Based Index of Biotic Integrity (FIBI) for Monitoring Riverine Ecosystems in the Lake Victoria Drainage Basin, Kenya. River Res. Applic. 28, 23-38.

Raven, P. J., Holmes, N. T. H., Dawson, F. H., Fox, P. J. A., Everard, M., Fozzard, I. R., Rouen, K. J. 1998: River habitat quality, the physical character of rivers and streams in the UK and Isle of Man. River Habitat Survey 2. Bristol.

Rogers, A. 2016. Habitat Quality Index metrics and Calculations; Water Quality Program Texas Parks and Wildlife Department TCEQ Biological Monitoring Training. 1 - 79.

Sitoki, L., Gichuki, J., Ezekiel, C., Wanda, F., Mkumbo, O. and Marshall B. E. 2010. The environmental of Lake Victoria (East Africa) current status and history changes. Internat. Rev. Hydrobiol. 95. 2010.3.209-223. 
Sharma, A., Bora, C. R., \&Shukla, V. 2013.Evaluation of seasonal changes in physico-Chemical and bacteriological characteristics of water from the Narmada River (India) using multivariate analysis. Natural Resources Research, 22(4), 283-296.

Staniszewski, R., Szoszkiewicz, K., Zbierska, J., Lesny, J., Jusik, S., \& Clarke, R. T. 2006. Assessment of sources of uncertainty in macrophyte surveys and the consequences for river classification. Hydrobiologia, 566, 235-246.

Vannote, R.L., G.W. Minshall, K.W. Cummins, J.R. Sedell, and C.E. Cushing, 1980. The river continuum concept. Canadian Journal of Fisheries and Aquatic Sciences, 37: 130- 137.

Witte, F., and Densen, W. L. T. 1995.Fish stock and fisheries of Lake Victoria. In a handbook for field observations. Cardigan (UK), Samara publishing limited.

World Health Organisation (WHO). 2015. UN-Water Glaas'Trach Fin Initiative: Tracking financing to sanitation, hygiene and drinking water at the national level. Guidance document summary for decision-makers. Geneva Switzerland, (Author), pp. 3-19.

Ward, J. V. 1998. Riverine landscapes: biodiversity patterns, disturbance and aquatic conservation. In: Biological Conservation, 83, 269-278. 